\title{
Einsatz für die Menschenwürde - Die Arbeit in der Bundesstelle zur Verhütung von Folter
}

\section{Sarah Mohsen, E.MA}

Rechtsassessorin/Wissenschaftliche Mitarbeiterin der Bundesstelle zur Verhütung von Folter, Wiesbaden

Etwas mehr als 100 Tage liegt der Berufseinstieg bei mir nun schon zurück, aber auch meinen Arbeitsplatz gibt es erst seit etwas über einem Jahr. Die neu geschaffene Bundesstelle zur Verhütung von Folter, deren wissenschaftliche Mitarbeiterin ich seit letztem Jahr bin, hat erst am 1. Mai 2009 ihre Arbeit in Wiesbaden aufgenommen.

Die Bundesstelle zur Verhütung von Folter wurde in Umsetzung des von Deutschland unterzeichneten Fakultativprotokolls zu dem Übereinkommen der Vereinten Nationen gegen Folter und andere grausame, unmenschliche oder erniedrigende Behandlung oder Strafe gegründet. Das Fakultativprotokoll sieht die Einrichtung nationaler Mechanismen zur Verhütung von Folter vor. Diese nationalen Mechanismen sollen durch ein System präventiver Besuche den Schutz vor Folter und Misshandlung an Orten der Freiheitsentziehung verstärken. Sie haben die Aufgabe, jederzeit und ggf. unangekündigt solche Orte aufzusuchen, um auf Missstände aufmerksam zu machen und Verbesserungsvorschläge vorzulegen. In Deutschland handelt es sich vor allem um Justizvollzugsanstalten, Polizeidienststellen des Bundes und der Länder, psychiatrische Anstalten und Arresteinrichtungen der Bundeswehr. In die Zuständigkeit der Bundesstelle fallen allerdings nur die Gewahrsamseinrichtungen der Bundespolizei und der Bundeswehr. Für Einrichtungen im Zuständigkeitsbereich der Bundesländer wird eine noch in diesem Jahr einzurichtende Länderkommission zur Verhütung von Folter zuständig werden. Organisatorisch ist die Bundesstelle, wie auch künftig die Länderkommission, der Kriminologischen Zentralstelle e.V. (KrimZ), einer kriminologischen Forschungs- und Dokumentationseinrichtung des Bundes und der Länder, in Wiesbaden angegliedert.

Oft werde ich gefragt, wie ich als Volljuristin überhaupt zu diesem Dienstposten gekommen bin? Durch mein Studium mit völkerrechtlichen Bezügen sowie die verschiedenen Studien- bzw. Praktikumsaufenthalte im Ausland bin ich schon früh in den Nischenbereich „Menschenrechte“ vorgedrungen. Da lag es nur nahe, nach dem 2. Staatsexamen auch eine Tätigkeit mit einem Menschenrechtsbezug anzustreben. Ich erinnere mich noch sehr gut an die ersten Tage, Wochen und Monate der Stellensuche - das war schon sehr frustrierend. Schließlich gibt es nur sehr wenige Stellenausschreibungen im Menschenrechtsbereich. Drei Viertel der in Betracht kommenden Posten werden zudem im Ausland angeboten, vor allem durch die Vereinten Nationen und den Europarat. Aber auch verschiedene NGOs sind hier erste Anlaufstellen. Dabei ich musste feststellen, dass die Bewerbungs- und Auswahlmühlen bei internationalen Organisationen sehr langsam mahlen. Da kann es schon mal sechs bis acht Monate dauern, bis man eine endgültige Rückmeldung auf die Bewerbung erhält. Insofern musste ich lernen, mich in Geduld zu üben.

Als ich dann auf das Stellenangebot der Kriminologischen Zentralstelle gestoßen bin, war ich erst mal einigermaßen überrascht, dass eine solche Stelle in Deutschland eingerichtet werden soll. Deutschland macht ja glücklicherweise nicht gerade als „Folterstaat" von sich reden und was wollte man da folglich überhaupt verhüten? Andererseits leuchtet ein, dass die Verpflichtung aus einem völkerrechtlichen Vertrag alle Parteien gleichermaßen betrifft, seien sie nun Leuchttürme der Rechtsstaatlichkeit oder aber sogenannte Schurkenstaaten.

Der besondere Reiz dieser Stelle liegt für mich vor allem darin, dass ich als wissenschaftliche Mitarbeiterin den Aufbau einer komplett neuen Einrichtung aktiv mitgestalten und begleiten kann. Die ersten 100 Tage in meinem neuen „Amt“ stellten mich demnach vor eine Vielzahl von Herausforderungen, zu deren Bewältigung mir meine juristischen Kenntnisse zunächst wenig nutzten. Da waren vielmehr Kreativität, Organisationstalent und Improvisation gefragt. Es mussten eine Webseite entworfen, die Außendarstellung der Bundesstelle entwickelt sowie Kontakte zu Ministerien und anderen Behördenvertretern, NGOs, internationalen Organisationen und anderen nationalen Präventionsmechanismen aufgebaut werden.

Weiterhin ging es darum, sich einen Überblick über die bestehenden Einrichtungen der Freiheitsentziehung und die jeweiligen Rechtsgrundlagen zu verschaffen. Schließlich wurden Arbeitsrichtlinien, Besuchsablaufpläne, Checklisten und Fragenkataloge für Inspektionsbesuche entworfen. Gemeinsam mit dem Leiter der Bundesstelle fanden diverse Sitzungen in Berlin und Wiesbaden statt, bei denen die Eckpunkte der künftigen Tätigkeit festgelegt wurden. Erst als diese Vorarbeiten abgeschlossen waren, konnten wir mit unserer eigentlichen Kerntätigkeit, der Durchführung von Inspektionsbesuchen bei Bundespolizei und Bundeswehr, beginnen.

Wie aber muss man sich diese Inspektionen vorstellen, und was genau ist meine Aufgabe bei der Durchführung der Besuche? Inspektionsbesuche können angekündigt oder auch unangekündigt stattfinden. Der Leiter der Bundesstelle und ich suchen die ausgewählten Dienststellen gemeinsam auf. Der Fokus liegt dabei zum einen auf den Gewahrsamsbereichen, aber auch auf dem menschenwürdigen Umgang mit den Personen in Gewahrsam insgesamt. Es werden alle Gewahrsamsbereiche inspiziert, außerdem finden Gespräche mit der Dienststellenleitung, ihrem Mitarbeiterstab sowie vertrauliche Gespräche mit einzelnen Personen in Gewahrsam statt. Anschließend werden die Ergebnisse des Besuchs in einem Bericht festgehalten und sich daraus ableitende Empfehlungen an das jeweils zuständige Ministerium abgefasst. Mir fällt 
dabei die entscheidende Rolle bei der inhaltlichen Vor- und Nachbereitung der Besuche zu. Meine juristischen Fähigkeiten sind vor allem bei rechtlichen Recherchen gefragt (z.B. die menschenrechtlichen bzw. grundrechtlichen Anforderungen an die Ausstattung von Gewahrsamszellen; Umfang bzw. Inhalt rechtlicher Belehrungen usw.).

Nach nun mehr als einem Jahr kann ich rückblickend sagen, dass es sich wirklich gelohnt hat, ein wenig länger nach der richtigen Stelle zu suchen. Ich bearbeite ein sehr vielfältiges Aufgabengebiet, das auf spannende Weise das nationale mit dem internationalen Recht verknüpft. Außerdem komme ich ständig in Kontakt mit interessanten Menschen, lerne viele staatliche Bereiche und auch deren Probleme kennen. Zu guter Letzt will ich auch erwähnen, dass meine Tätigkeit mit vielen Dienstreisen (auch ins Ausland) verbunden ist - eine Tatsache, die ebenso meinen Neigungen entspricht. Letztlich möchte ich andere junge Juristinnen ermutigen, sich bei der Stellensuche in einem so speziellen Bereich ruhig ein wenig Zeit zu lassen. Ich bin mir sicher, dass das Warten am Ende auch mit einer überaus reizvollen, abwechslungsreichen und erfüllenden Tätigkeit belohnt wird.

\section{Arbeitsauftrag: Art. 3 II 2 GG - Universitäre Gleichstellungsarbeit}

\section{Lea Junghans \\ Rechtsassessorin/Wissenschaftliche Mitarbeiterin an der Universität Köln}

Bereits in der letzten Referendariatsstation war ich bei der Gleichstellungsbeauftragten der Uni Köln. Danach hatte ich die Wahl: als Juristin in eine städtische Verwaltung zu wechseln oder als wissenschaftliche Mitarbeiterin bei der Gleichstellungsbeauftragten zu bleiben. So ganz leicht war die Entscheidung nicht, denn ich hatte große Bedenken, mich zu sehr „vom Juristischen“ zu entfernen. Auf der anderen Seite stand die Tatsache, dass es - zumindest für mich - einen Sechser im Lotto bedeutete, mit „Frauenrechten“ Geld zu verdienen. Bereits im Studium saß ich in Vorlesungen der Gender Studies, hatte meine Wahlfach-Seminararbeit über den Gender-Mainstreaming-Prozess geschrieben und in der Anwaltsstation monatelang einen „Kopftuchstreit" sowie andere Diskriminierungsfälle bearbeitet und bin auch privat in diesem Bereich unterwegs.

Da ich keine Erfahrungen in der Projektarbeit, in der Wissenschaftsverwaltung oder der professionellen Gleichstellungsarbeit hatte, gab es in den ersten drei Monaten erst einmal nebenberuflich an der Uni Rostock einen Fernkurs „Qualitätsmanagement in der Öffentlichen Verwaltung “. So kurz nach dem zweiten Staatsexamen Klausuren und die nächste Abschlussarbeit („Qualitätsentwicklung in den Tätigkeiten der Gleichstellungsbeauftragten“) zu schreiben, war ziemlich ernüchternd. Aber Fortbildungen und interdisziplinäre Kenntnisse sind in alternativen juristischen Berufen unabdingbar; gerade auch im Bereich der „Gender Studies“ ist der wissenschaftliche Anspruch nicht zu unterschätzen.

Über mangelnden juristischen Bezug konnte ich mich jedoch nicht besonders beschweren; so ziemlich jedes Rechtsgebiet hatte ich schon zu bearbeiten. Gerade in den ersten drei Monaten kam ich mir vor wie eine „Feld-Wald-und-Wiesen-Anwältin“. Wie sicherlich jede Juristin in einem Büro voller Nichtjuristinnen musste ich erst einmal nach und nach klar machen, dass es zwar den „Einheitsjuristen " gibt, aber ich mit meinem Schwerpunkt im Verwaltungs- und Gleichstellungsrecht mir nicht jede Antwort zu Entfristungsklagen, Mutterschutz und Strafrecht aus dem Ärmel schütteln und eine Einarbeitung bei Eventualfragen auch unverhältnismäßig sein kann; zumal es in den Dezernaten hoch spezialisierte Verwaltungsjurist(inn)en gibt. Gerade in einem unabhängigen Organ kann man jedoch die anderen juristischen Kompetenzen der Einrichtung nicht immer in Anspruch nehmen oder muss sich gerade mit diesen um die richtige Gesetzesanwendung streiten. Bereits in den ersten drei Monaten führte ich seitenlange Auseinandersetzungen mit Jura-Professoren über das Landesgleichstellungsgesetz. In traditionellen juristischen Berufen sollte man sicherlich nicht besonders harmoniesüchtig sein; in der Gleichstellungsarbeit jedoch noch viel weniger. Wenn in meiner Arbeit das Gesetz ins Spiel kommt, ist es höchst selten, dass ich nicht zeitgleich den Sinn und die Legitimationen der entsprechenden Normen erklären muss - Gesetz und Rechtsprechung sind selten ausreichende Argumente. Zudem ist das Amt der Gleich- 